\title{
ANGIOMATOSIS PÉLVICA COMO CAUSA DE HEMATURIA
}

\author{
M.P. PÉREZ ESPEJO, P. CAMPOY MARTÍNEZ, D. CHINCHÓN ESPINO, \\ F.J. RODRÍGUEZ CORCHERO, M. PÉREZ PÉREZ, P. MONTAÑÉS MEDINA
}

Servicio de Urología. Hospital Universitario Virgen del Rocío. Sevilla.

Actas Urol Esp. 28 (6): 462-465, 2004

\section{RESUMEN}

ANGIOMATOSIS PÉLVICA COMO CAUSA DE HEMATURIA

Los angiomas renales (también llamados hemangiomas) son malformaciones vasculares congénitas (consideradas como tumores benignos), de etiología no conocida y de escasa traducción clínica (hematuria y cólico ureteral asociado al paso de coágulos por la vía urinaria).

OBJETIVO: Presentar un caso de hematuria unilateral severa en mujer joven sin antecedentes destacables, secundaria a hemangioma renal pélvico izquierdo.

MATERIAL Y MÉTODOS: Caso clínico de mujer de 16 años, sin antecedentes personales de interés, que presenta hematuria anemizante con coágulos de dos meses de evolución. Como técnicas diagnósticas se utilizaron ecografía, urografia intravenosa, cistoscopia, TAC, angiografía, y gammagrafía. Para su tratamiento precisó de intervención quirúrgica (nefrectomía lumbar).

RESULTADO: Anatomía patológica de riñón con proceso proliferativo de tipo vascular submucoso en pelvis renal, compuesto de vasos venosos y compatible con hemangiona.

PALABRAS CLAVE: Hematuria. Hemangioma renal.

\section{ABSTRACT \\ KIDNEY PELVIS HEMANGIOMA AS A CAUSE OF HEMATURIA}

Kidney hemangiomas are congenital vascular anomalies with an unknown ethiology. They are considered as benign tumours with a poor clinic expression (hematuria or ureteric colic, which is associated to the drop of clots in the urinary tract.

OBJECTIVE: To expound a case of unilateral severe hematuria in a young woman with a restless past medical history, due to a pelvic left kidney hemangioma.

MATERIALS AND METHODS: Clinic case of a sixteen years old woman, no relevant past medical history, presented with an anaemizing gross hematuria and passing vermiform clots.

As diagnostic techniques we used ultrasonography, excretory urography, cystourethroscopy, CT, angiography and nuclear imaging. Surgery (flank nephectomy) was needed for treatment.

RESULTS: Pathology report: submucosal vascular proliferative process in kidney pelvis composed by blood vessels, suggesting haemangioma.

KEY WORDS: Hematuria. Kidney hemangioma.

$\mathrm{L}^{\mathrm{a}}$ a hematuria puede convertirse en ocasiones en un difícil y delicado proceso diagnóstico urológico. Radiólogos, médicos nucleares, urólogos y otros especialistas deben aunar esfuerzos para conseguir un diagnóstico certero en casos especiales $^{1}$.
La hematuria unilateral esencial, que se describe como un sangrado unilateral, crónico, de origen indeterminado, en ocasiones no se puede diagnosticar con todos los métodos radiológicos y clínicos a nuestro alcance. Sólo el examen cistoscópico podrá demostrar que la hematuria es 
unilateral, sin embargo, encontrar la localización exacta; y más aún, la causa, es en muchas ocasiones imposible ${ }^{2}$.

Aunque el tratamiento debe ser individualizado para cada paciente, los primeros pasos son claros: ecografía, citología, urografía intravenosa y/o TAC y cistoscopia ${ }^{3}$. Los avances en instrumentos endourológicos han cambiado drásticamente el manejo de estos enfermos con lesiones en el aparato urinario superior, y así, la ureteropieloscopia, que ha sido múltiples veces ensayada con éxito en el tratamiento de los cálculos ureterales, ha alcanzado un valor incalculable para el diagnóstico y a veces tratamiento de lesiones pieloureterales ${ }^{4}$.

Presentamos un caso de hematuria unilateral severa, de difícil diagnóstico. El minucioso proceso seguido hasta su curación, así como la florida iconografía, nos ha llevado a publicarlo.

\section{CASO CLÍNICO}

Mujer de 16 años de edad, sin antecedentes personales de interés que consulta por hematuria de dos meses de evolución, con coágulos alargados, indolora, anemizante, con requerimiento transfusional.

La ecografia fue normal, la citología urinaria mostró hematíes, displasia moderada y flora mixta coco-bacilar. Se practicó urografía intravenosa que fue rigurosamente normal.

Se decide realizar cistoscopia a la paciente, encontrando una vejiga normal y eyaculación hematúrica por meato ureteral izquierdo.

A continuación se realiza TAC con y sin contraste, encontrando una lesión hipodensa en pelvis renal izquierda, que desaparece tras la inyección de contraste (Figs. 1 y 2).

La siguiente prueba que se practica es una arteriografía segmentaria abdominal con arteriografía selectiva renal izquierda, siendo normal la vascularización, el tiempo nefrográfico y el tiempo venoso.

Tras consulta con el Servicio de Medicina Nuclear, se realiza en esta ocasión gammagrafía con hematíes marcados con Tecnecio-99, sin que tampoco se logre descubrir la causa de la hematuria (Fig. 3).

Durante todo este periodo, la paciente continúa con hematuria continua, de mediana cuan-

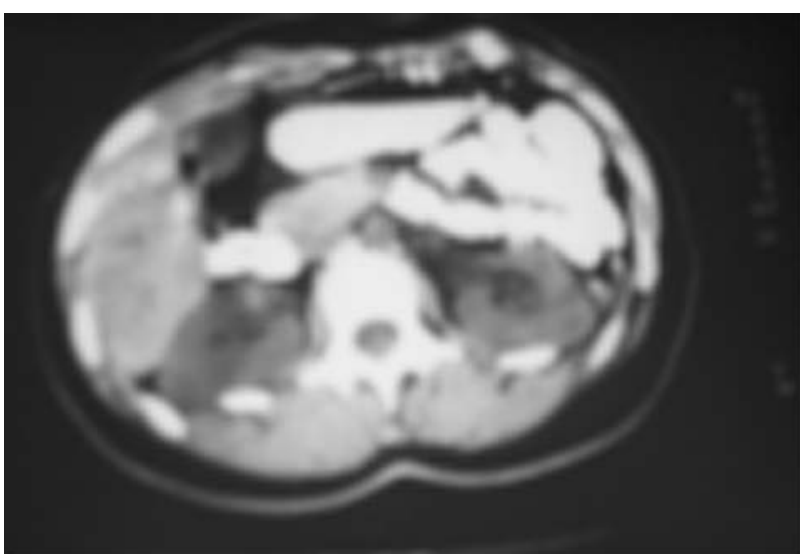

FIGURA 1. TAC sin contraste que objetiva una lesión hipodensa en pelvis renal izquierda.

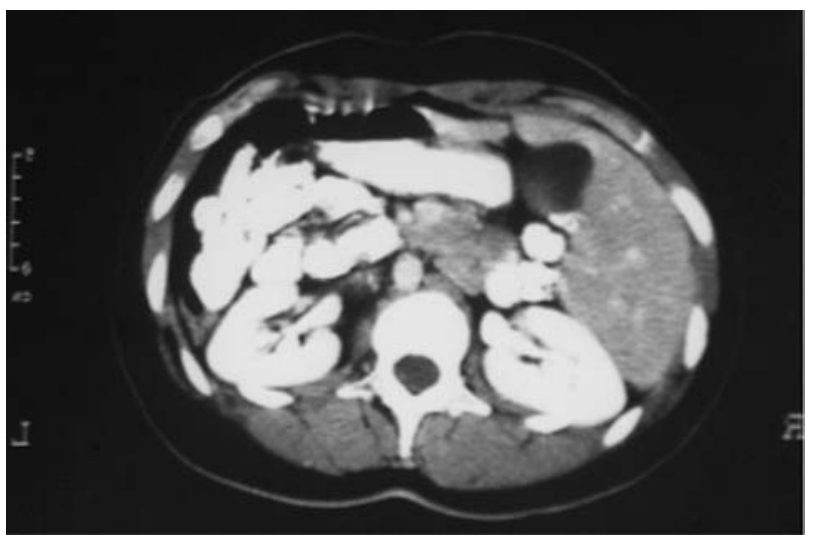

FIGURA 2. TAC en que se objetiva la desaparición de la lesión pélvica renal izquierda tras la inyección de contraste.

tía, precisando nuevas transfusiones por lo que se plantea una actitud más agresiva, realizando bajo anestesia general, ureteroscopia izquierda visualizando un uréter normal, con coágulo molde en pelvis renal, no encontrando la causa del sangrado. Se coloca a la paciente en posición de lumbotomía y tras apertura de pelvis renal y evacuación del coágulo, se encuentra un riñón macroscópicamente normal y se practica nefrectomía simple.

La paciente cursó un post-operatorio sin complicaciones, estando diez años después asintomática.

La anatomía patológica fue informada como riñón con proceso proliferativo de tipo vascular submucoso en pelvis renal, compuesto de vasos venosos y compatible con hemangioma (Fig. 4). 


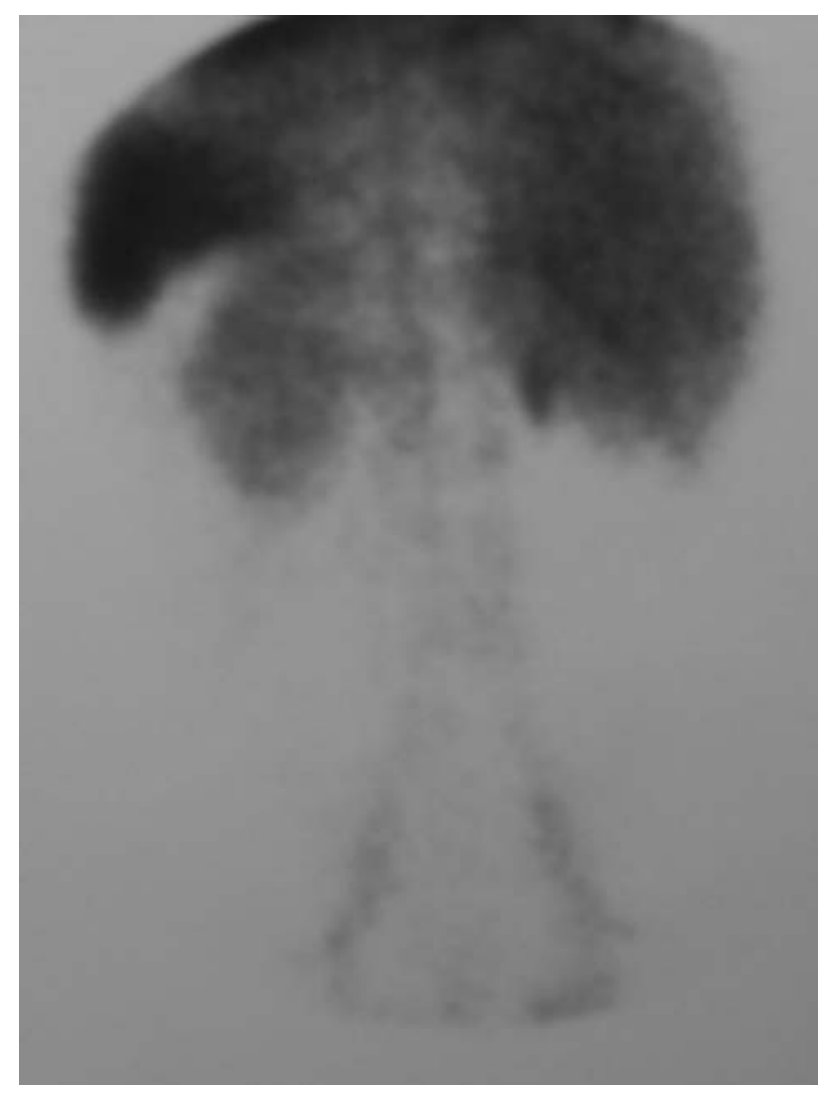

FIGURA 3. Gammagrafia con hematies marcados con Tc99, en que no se objetiva foco de captación patológica.

\section{DISCUSIÓN. CONCLUSIONES}

Fenwick describió en 1904 los seis primeros casos de hemangioma renal; a partir de aquí y gracias a las nuevas técnicas diagnósticas, se han descrito hasta la actualidad multitud de nuevos casos, la mayoría etiquetados previamente como hematuria esencial ${ }^{1}$.

Estas malformaciones vasculares congénitas son de etiología no conocida, y de escasa traducción clínica, y son consideradas como tumores vasculares renales benignos, y causa de hematuria renal poco frecuente ${ }^{5}$.

En la mayoría de las ocasiones son asintomáticos y se encuentran incidentalmente durante el curso de una autopsia. Los infrecuentes signos de un hemangioma renal son hematuria, y debido a este cólico ureteral asociado al paso de coágulos por la vía urinaria $(18-41 \%$ de los pacientes) ${ }^{6}$.

Aproximadamente el 12\% de los hemangiomas son múltiples, pero menos del $1 \%$ son bilaterales.

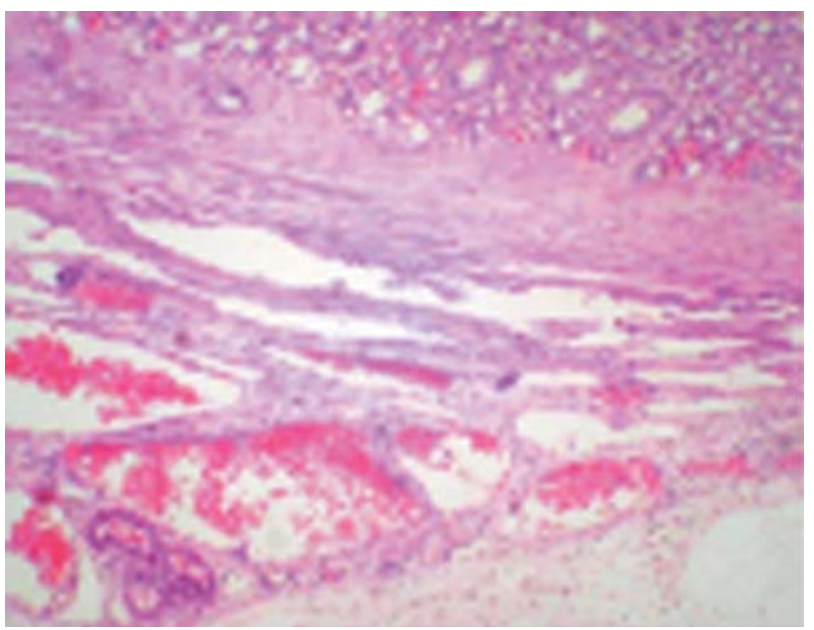

FIGURA 4. Proceso proliferativo submucoso vascular renal, compuesto por vasos sanguíneos, sugerente de hemangioma.

El hemangioma renal puede ser una lesión aislada o formar parte de un síndrome congénito, como el Sturge-Weber, la angiomatosis sistémica, o el síndrome de Klippel-Trenaunay ${ }^{7}$.

Cronológicamente, su máxima incidencia corresponde a la tercera y cuarta década de la vida; no predomina en sexo, raza ni lateralidad respecto a ambos riñones.

La clasificación de los hemangiomas es en papilares, cavernosos, mixtos y arterio-venosos. Suelen estar en la médula renal, cerca de los cálices, o en la submucosa de las papilas renales, menos frecuentemente en la pelvis, subcapsulares o dentro de un quiste renal ${ }^{8}$.

El diagnóstico preoperatorio no siempre es posible, debido principalmente al pequeño tamaño del tumor. Suele seguir la metodología diagnóstica del síntoma más prevalente, es decir, la hematuria. Difícilmente será realizado por métodos exploratorios convencionales ${ }^{2}$. De esta forma las ecografias y urografías endovenosas son normales en el $50 \%$ de los casos ${ }^{1}$, sólo nos demostrarán hallazgos patológicos en caso de hemangiomas de gran tamaño (defectos de repleción e irregularidades en la silueta de los cálices). La cistoscopia evidenciará el carácter unilateral de la hematuria ${ }^{5}$. La arteriografia es desde hace algunas décadas el procedimiento diagnóstico de elección; aún así un alto porcentaje de ellas no logra establecer la localización del hemangioma ${ }^{3}$. Cuando la arteriografía renal muestra la lesión, el 
tratamiento de elección es la embolización, siempre y cuando sea posible y seguro. La gammagrafía con hematies marcados con Tecnecio-99 puede ayudarnos a concretar el origen del sangrado, con vistas a una cirugía parcial ${ }^{6,8}$. Esta técnica objetiva zonas de captación tardía del radionúclido en la zona correspondiente al hemangioma. Sin embargo, las nuevas técnicas diagnósticas como nefroscopia anterógrada y ureteropieloscopia retrógrada la hacen menos relevante. Mediante las mismas se pueden objetivar lesiones sospechosas en papilas renales, de las que se tomarán biopsias, o bien detectarse focos de hemorragia babeante procedentes de alguna papila renal, identificables tan sólo al interrumpir el sistema de irrigación continua del instrumental endoscópico y que permite la toma de biopsias dirigidas, la electrocoagulación o laserificación del área biopsiada ${ }^{4,9}$.

En nuestro caso ninguna de las exploraciones anteriores definió la región renal patológica, y en estos casos, y ante el riesgo de inestabilidad hemodinámica de la paciente, la nefrectomía, una vez establecida con seguridad la unidad renal sangrante, es la opción más razonable ${ }^{7}$.

Algunos autores han ensayado la instilación de fármacos hemostáticos y la radioterapia, con resultados desiguales. Estas técnicas actualmente están abandonadas.

El pronóstico, una vez localizada y extirpada la lesión, es excelente, habida cuenta de que la bilateralidad es muy rara.

\section{REFERENCIAS}

1. PUIGVERT A.: Hemangiomas urológicos. Arch Esp Urol 1984; 37: 275-279.

2. CARTER WC, ROSS SN.: Gross haematuria in 110 adult urologic hospital patients. Urology 1981; 18: 342-344.

3. MARIANI AJ, MARIANI MC, MACCHIONI C, STAMS UK, HABIHARAN A, NORIERA A.: The significance of adult haematuria: 1000 haematuria evaluations including a risk-benefict and cost-effectiveness analysis. $J$ Urol 1989; 141: 350-354.

4. KUMON H, TSUGAWA M, MATSUMURA Y, OHMORI H.: Endoscopic diagnosis and treatment of chronic unilateral haematuria of uncertain etiology. $J$ Urol 1990; 143: 554-558.

5. LAUCIRICA O, IZQUIERDO F, MARTI J, LAGUNA P, PALOU J, VICENTE J.: Hemangiomas renales: caso clínico y revisión de la literatura. Actas Urol Esp 1992; 16: 366-370.

6. FERRE J, SANJUÁN C, VILAR J, VILLANUEVA A, BAEZA R.: Hemangioma of the kidney. Eur Urol 1983; 9: 189-192.

7. FLIGELSTONE LJ, CAMPBELL F, RAY DK, REES RWM.: The Klippel-Trenaunay syndrome: a rare cause of haematuria requiring nephrectomy. J Urol 1994; 151: 404-405.

8. SAMELLAS W, MORPHIS LG, BAKOPOULOS CV.: Hemangioma of the kidney. J Urol 1976; 116: 653654.

9. TAWFIEK ER, BAGLEY DH.: Ureteroscopic evaluation and treatment of chronic unilateral haematuria. J Urol 1998; 160: 700-702.

Dra. $\mathrm{M}^{\mathrm{a}}$ de la Paz Pérez Espejo

C/ Cervantes, 17 - 2o Dcha

41003 Sevilla

(Trabajo recibido el 25 julio de 2003) 